\title{
Nano-motion control of heavy quadrupoles for future particle colliders: An experimental validation
}

\author{
C. Collette*, S. Janssens, K. Artoos, A. Kuzmin, P. Fernandez-Carmona, M. Guinchard, \\ R. Leuxe, C. Hauviller \\ Engineering Department, European Organization for Nuclear Research, Geneva 23, 1211 Geneva, Switzerland
}

\section{A R T I C L E I N F O}

\section{Article history:}

Received 6 January 2011

Received in revised form

11 April 2011

Accepted 16 April 2011

Available online 27 April 2011

Keywords:

Quadrupole adaptive repositioning

Seismic vibration isolation

Active stabilization

Nano-positioning

\begin{abstract}
A B S T R A C T
This paper presents an experimental validation of a control strategy capable of both stabilizing and positioning the heavy electromagnets of future particle colliders. The originality of the approach is to use the same active mounts to perform both tasks, with a nanometer precision. In a previous paper, the concept has been studied numerically, and validated on a scaled single degree of freedom (d.o.f.) test bench. In this paper, it is extended to a two d.o.f. test bench, constituted of a heavy mass mounted on two active legs. Firstly, the model is described and the performances are discussed numerically. Secondly, experimental results are presented, and found to correlate well with the model, and comply with the requirements. Finally, the experimental results are combined with a simplified model of the beam-based feedback to evaluate the jitter of the beam. It is found that, at the scale of a single quadrupole, the mechanical stabilization of the quadrupoles reduces the vertical beam jitter by a factor 10 .
\end{abstract}

(c) 2011 Elsevier B.V. All rights reserved.

\section{Introduction}

The objective of the future Compact LInear Collider (CLIC) currently under study [1] is to collide two beams of particles (electrons and positrons) at an energy of $0.5-3 \mathrm{TeV}$. It has been estimated that the required luminosity of $5.9 \times 10^{34} \mathrm{~cm}^{-1} \mathrm{~s}^{-1}$ implies that the beam should be stable at the nanometer scale [1]. To reach this challenging objective, the electromagnets (quadrupoles) used to focus the two beams have to fulfill a certain number of requirements. In Ref. [2], an original concept has been introduced to fulfill all of these requirements using the same active support. It is based on the use of inclined active legs [3-5], and an inertial reference. This allows to combine a good robustness to external forces and the capability to stabilize or move the quadrupole in both the vertical and the lateral directions. The strategy is also easily adaptable to the four different types of quadrupoles of the CLIC. The concept has been studied numerically, and validated on a scaled single degree of freedom (d.o.f.) test bench [2]. In this paper, it is extended to a two d.o.f. test bench, constituted of a heavy mass mounted on two active legs. As announced in Ref. [2], the objective of this second test bench is to address most of the issues in the control of the heaviest, slender CLIC quadrupoles. This paper presents the results of this new important milestone and is organized as follows. Section 2

\footnotetext{
* Corresponding author.

E-mail address: christophe.collette@cern.ch (C. Collette).
}

presents in details the requirements for the supports and the design constraints. Section 3 presents the model of the test bench and the numerical results. Section 4 presents the experimental results. Section 5 evaluates, with a simple model, the effect of the mechanical stabilization on the jitter of the beam. Section 6 draws the conclusions.

\section{Requirements}

- The quadrupoles have to be extremely stable. Let $\Phi_{x}(f)$ be the power spectral density of the vertical displacement of the quadrupole. It has been estimated that the integrated Root Mean Square (RMS) $\sigma_{x}(f)$, defined as

$\sigma_{x}(f)=\sqrt{\int_{f}^{\infty} \Phi_{x}(v) d v}$

must stay below $1.5 \mathrm{~nm}$ [6] above $1 \mathrm{~Hz}$ in order to minimize the emittance. Similarly, it must stay below $5 \mathrm{~nm}$ in the lateral direction. This concerns about 2000 quadrupoles per beam line.

- About 80 of these quadrupoles should have the capability to move by steps of some tens of nanometers every $20 \mathrm{~ms}[7,8]$, with a precision of $\pm 1 \mathrm{~nm}$, in the vertical and the lateral direction.

- The size of the tunnel is very restricted, and the space available for the mounts should not exceed a height of $15 \mathrm{~cm}$. 
- The direct environment of the future CLIC collider is subjected to radiations and stray magnetic fields. In order to ensure a full compatibility with this environment, this requirement excludes the use of electromagnetic equipment (electromagnetic actuators and sensors using coils likes commercial seismometers).

- The quadrupoles are pre-aligned by an alignment system that is located under the stabilization system. This pre-alignment is achieved with a micrometer precision. The stabilization system should be compatible with this alignment system, i.e. it should not alter the pre-alignment. This means that the stabilization system should be stiff, and that the displacements imposed by the alignment system should be precisely measured.

- In operating conditions, the quadrupoles are also subjected to several types of disturbances, commonly referred to as technical noise: acoustic noise, cooling system, ventilation. The supports should accordingly ensure a sufficient robustness to the external forces generated by these disturbances.

- The support should allow a temperature change of about $25 \mathrm{~K}$ during transients. Features should be implemented to protect the actuating system during transport and handling.

A priori, the first two requirements are conflicting [2]. The first one suggests to use a soft support, in order to benefit from the passive isolation at high frequency. The second one requires to mount the quadrupole on strong actuators, to allow a fast positioning at $50 \mathrm{~Hz}$ of the heavy mass, and also to ensure a sufficient robustness to technical noise. In order to comply with all of these requirements using a unique support, an original concept, based on inclined active legs, has been introduced. In this paper, it is applied to a test bench, presented in the next section.

\section{Theoretical model of the test bench}

\subsection{Dynamics}

The test bench is inspired from Refs. $[9,10]$. However, it is designed to be modular, in order to be able to address a certain number of difficulties including: the stabilization in both vertical and lateral direction, the nano-positioning in both vertical and lateral direction, mounting, jointure and guide design.

It is constituted of a compact mass of $m=100 \mathrm{~kg}$, supported on one side by two passive mounts and on the other side by two active mounts. A simplified three-dimensional sketch is shown in Fig. 1. In the hypothesis of small displacements, the two passive supports work as a guide to leave only two d.o.f. on the active side (vertical and lateral). Each active mount is composed of a piezoelectric stack actuator, mounted with two flexible joints to avoid backlash and friction. Assuming for simplicity that the rotation of the mass is perfectly blocked by the two passive mounts, the test bench can be approximated by the two d.o.f. model shown in Fig. 1(a).

Under this assumption, the dynamics of the system is governed by

$M \ddot{\mathbf{x}}=\mathbf{F}$

where $\mathbf{x}=(y, x)^{T}$ is a vector containing the lateral and vertical displacements of the mass, $M=\operatorname{diag}(m, m)$ is the mass matrix and $\mathbf{F}=\left(F_{y}, F_{x}\right)^{T}$ is the vector of forces applied by the legs on the mass, expressed in the coordinates of the mass.

Ignoring for simplicity the bending stiffness of the joints, $\mathbf{F}$ is related to the vector $\mathbf{f}=\left(f_{1}, f_{2}\right)^{T}$ of axial forces in each leg by

$\mathbf{F}=B \mathbf{f}$

where $B$ is the force Jacobian matrix. Assuming that there is no damping in the legs, $f_{i}$ is given by

$f_{i}=k_{a}\left(-q_{i}+\Delta_{i}+w_{i}^{l}\right)$

where $k_{a}$ is the axial stiffness of the leg, taken as $300 \mathrm{mN} / \mathrm{m}, q_{i}$ and $w_{i}^{l}$, are, respectively the displacement of the quadrupole and the ground in the direction of the leg. $\Delta_{i}$ is the elongation of the leg $i$ due to a voltage $V_{i}$ applied to the piezoelectric stack actuator, and

$\Delta_{i}=n d_{33} V_{i}$

where $n d_{33}$ is a characteristic of the actuator and $i=\{1,2\}$.

Replacing Eqs. (3) and (4) in Eq. (2) gives

$M \ddot{\mathbf{x}}+K \mathbf{x}=B \mathbf{u}+k_{a} B \mathbf{w}^{l}$

or again

$M \ddot{\mathbf{x}}+K \mathbf{x}=B \mathbf{u}+k_{a} B E \mathbf{w}$

where $K=k_{a} B B^{T}$ is the stiffness matrix, $\mathbf{w}$ and $\mathbf{w}^{l}$ are the ground excitation vector and the ground excitation vector in the directions of the legs, and $E$ is the excitation matrix projecting $\mathbf{w}$ in the directions of the legs. $\mathbf{u}=\left(u_{1}, u_{2}\right)^{T}$ is the vector of control forces, where $u_{i}=k_{a} \Delta_{i}$.

Using the virtual work principle, it can be shown (see Ref. [2]) that the Jacobian matrix, $J$, relating the elongations velocities of

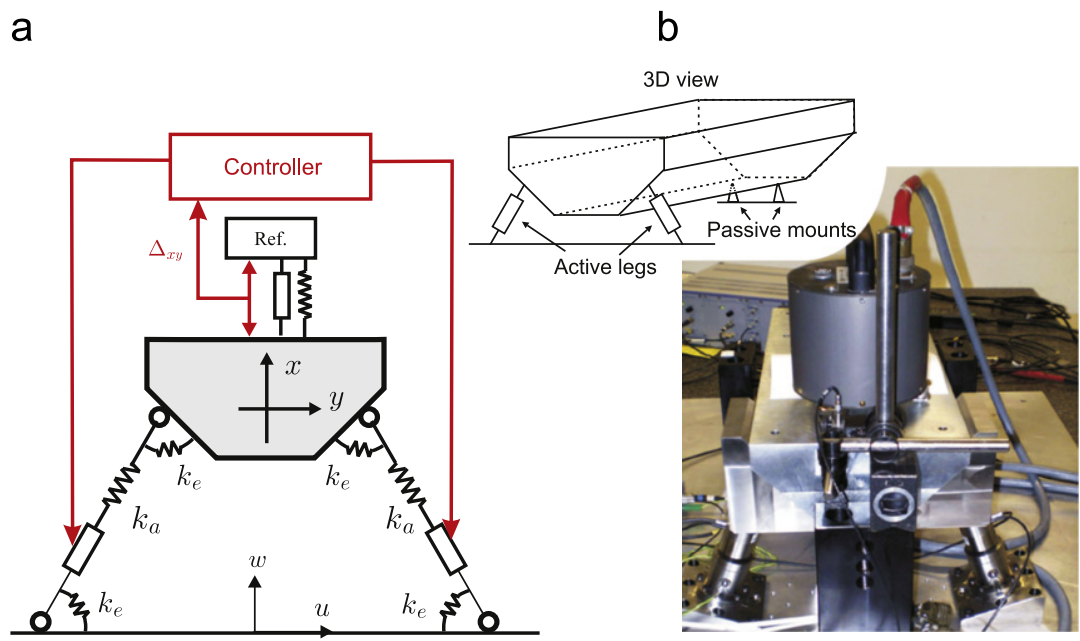

Fig. 1. (a) Model and (b) picture of the two d.o.f. test bench. 
the two legs $\dot{\mathbf{q}}=\left(\dot{q}_{1}, \dot{q}_{2}\right)$ and the velocity vector $\dot{\mathbf{x}}$ as $\dot{\mathbf{q}}=J \dot{\mathbf{x}}$ is given by

$J=\left(\begin{array}{cc}\sin \beta & \cos \beta \\ -\sin \beta & \cos \beta\end{array}\right)$

where $\beta$ is the inclination of the legs with respect to the vertical, and the other matrices are

$K=2 k_{a}\left(\begin{array}{cc}\sin ^{2} \beta & 0 \\ 0 & \cos ^{2} \beta\end{array}\right) ; \quad E=J=B^{T}$

\subsection{Stabilization}

The general idea applied to stabilize the quadrupole is based on the measurement of the relative displacement between the quadrupole and an inertial reference. The reference can be either on the quadrupole $[11,12]$ or on the ground [13-16]. For simplicity, the first option has been adopted in this work, and a seismometer has been used for the inertial reference. In the configuration described in Fig. 1(b), a seismometer is mounted on the quadrupole above the legs, and used as inertial reference. After integration, let $\mathbf{x}^{m}=\left(y^{m}, x^{m}\right)^{T}$ be the vector of the measured displacements of the quadrupole, used in a local feedback loop. The forces exerted by the actuators on the quadrupoles $\mathbf{u}=\left(u_{1}, u_{2}\right)^{T}$ are given by

$\mathbf{u}=k_{a}\left(\begin{array}{l}\Delta_{1} \\ \Delta_{2}\end{array}\right)=-k_{a} H(s)\left(\begin{array}{cc}\sin \beta & \cos \beta \\ -\sin \beta & \cos \beta\end{array}\right)\left(\begin{array}{l}y^{m} \\ x^{m}\end{array}\right)$

where $H(s)$ is the compensator and $\beta$ is the inclination angle of the leg with respect to the vertical direction. The compensator includes the sensor dynamics, and can be a scalar or a vector, depending on the control objective in the vertical and the lateral direction.

The controller consists of a second order Butterworth high pass filter at $0.5 \mathrm{~Hz}$, a first order low pass filter at $1 \mathrm{~Hz}$ to integrate the signals measured by the seismometer, and a double lead at $30 \mathrm{~Hz}$ to improve the stability of the feedback loop. The same compensator is applied in the vertical and the lateral direction, and used to command the actuators after multiplication by the Jacobian matrix. Figs. 2(a) and (b) show the theoretical closed loop transfer function between the ground and the mass in, respectively, the lateral direction, $T_{S}^{L}(f)$, and vertical direction, $T_{S}^{V}(f)$.

\subsection{Nano-positioning}

Besides isolation, the supports of the quadrupoles should also provide some positioning capability to perform an adaptive repositioning of the main beam quadrupoles. In order to evaluate the capability to fulfill this requirement, the following experiment has been performed. First, the ground vibrations in the LHC tunnel, in both the vertical and the lateral direction, have been recorded synchronously by two seismometers, placed $108 \mathrm{~m}$ apart (details on the measurements can be found in Ref. [17]).

Then, the difference of the signals from the two seismometers has been integrated and sampled at $50 \mathrm{~Hz}$, corresponding to the CLIC repetition rate of the pules. The resulting time histories (with coordinates $\left(r_{y}(t)\right.$ and $r_{x}(t)$ in Fig. 3(a))) represent a typical signal measured by the Beam Position Monitors (BPM), mounted on the quadrupoles, assuming that they are directly fixed on the ground. Then, these histories are used as a target to follow by one quadrupole, in order to remain perfectly aligned with another one placed $108 \mathrm{~m}$ apart, as shown in Fig. 3(b). In this case, the systems works in open loop. Fig. 3(a) shows that the system can a

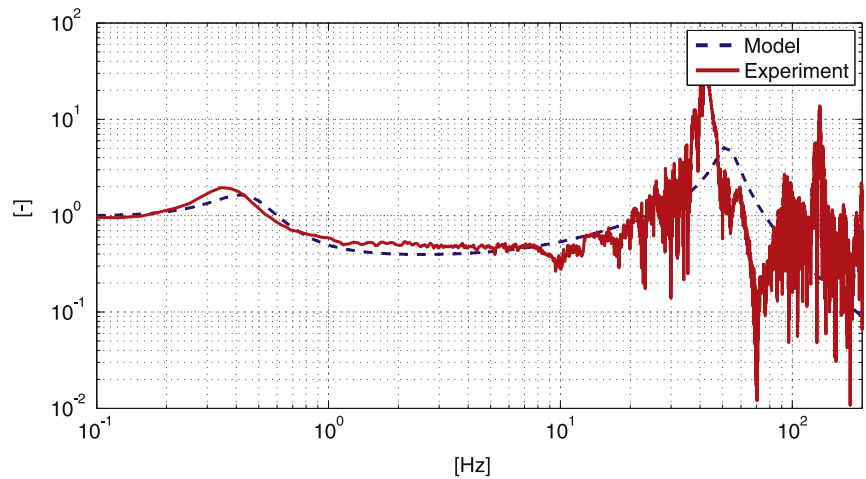

b

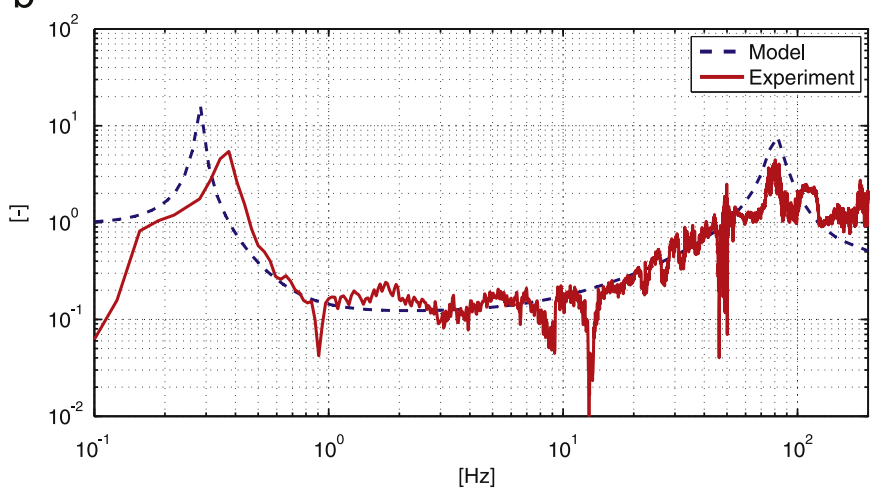

Fig. 2. Transmissibility between the ground and the mass of the test bench in the (a) lateral direction, $T_{S}^{L}(f)$, and (b) vertical direction, $T_{S}^{V}(f)$.

a

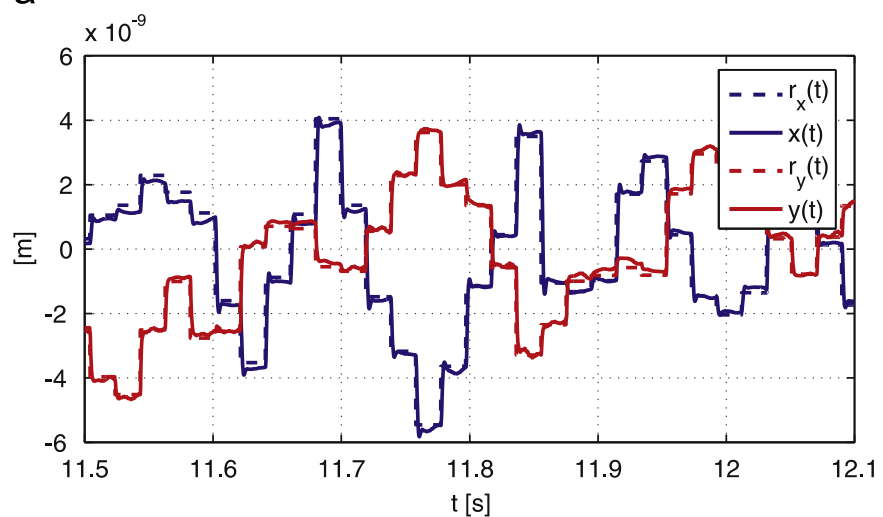

b

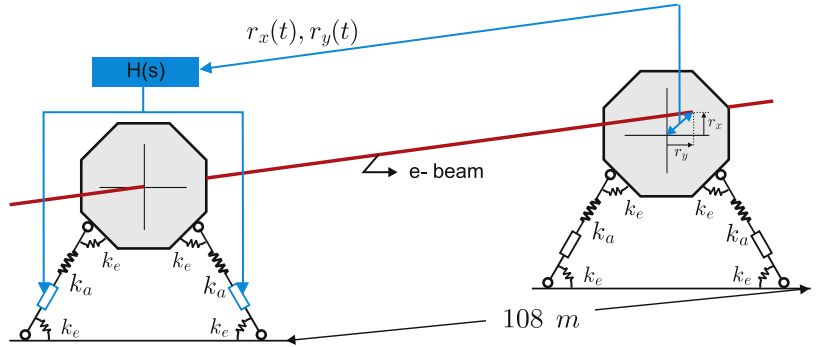

Fig. 3. (a) Simplified drawing of two quadrupoles separated by $108 \mathrm{~m}$. (b) Example of requested signal corresponding to the relative displacement of two points separated by $108 \mathrm{~m}$ in the LHC tunnel, sampled at $50 \mathrm{~Hz}$, and time histories of the model outputs $y(t)$ and $x(t)$ in open loop configuration.

follow the requested signal in both directions. The tracking capability is better in the vertical direction, because of the orientation of the legs. 


\section{Experiments}

The picture shown in Fig. 1(b) is a front view of the test bench, showing the compact mass mounted on the two active legs. These legs are basically constituted of high voltage piezoelectric stack actuators P-225.10 from Ref. [18], mounted with custom built flexible joints at both ends. One end is fixed on the mass, the other end is fixed on a straightened aluminum frame. The vibrations are measured with two seismometers CMG-6T from Ref. [19], one on the frame and one on the mass. Both of them measure the velocity in the vertical direction and the horizontal direction (perpendicular to the main axis of the mass). The real time digital control system is using a card PXI- 6289 from Ref. [20].

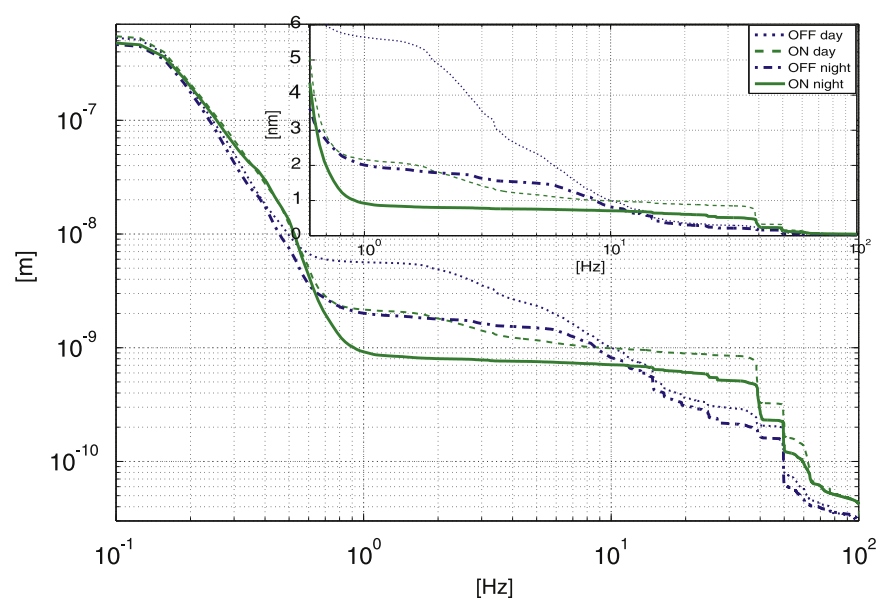

Fig. 4. Comparison of integrated RMS of the top displacement $\sigma_{x}(f)$, for the test bench 2 , when the controller is $\mathrm{ON}$ and $\mathrm{OFF}$, during the day and during the night.
The test bench is placed in a tunnel (ISR-I8) where the amplitude of the ground motion is similar to the values measured in the LHC tunnel. Day and night variations allow to study the test bench with a background varying between 2 and $7 \mathrm{~nm}$ vertical integrated RMS at $1 \mathrm{~Hz}$. The two following sections present, respectively, the stabilization and nano-positioning experimental performances of the test bench in this environment.

\subsection{Stabilization}

Figs. 2(a) and (b) show the measured transmissibility between the ground and the mass, respectively, in the lateral and vertical direction. These results show that the feedback operation reduces the transmitted seismic vibrations by a factor 2 in the lateral direction, and by a factor of nearly 10 in the vertical direction.

Fig. 4 shows the corresponding integrated RMS vertical displacement of the top seismometer for two experiments, one performed during the day and one performed during the night. During the day, at $1 \mathrm{~Hz}$, one sees that the feedback control has reduced $\sigma_{x}(f)$ from 5.7 to $2.1 \mathrm{~nm}$, i.e. a reduction by a factor 2.5 . During the night, when the ground motion is even lower, $\sigma_{x}$ is reduced from 2 to $0.9 \mathrm{~nm}$ at $1 \mathrm{~Hz}$, and $0.7 \mathrm{~nm}$ at $4 \mathrm{~Hz}$.

\subsection{Nano-positioning}

In order to test the nano-positioning capability of the test bench, two time histories have been used to command the two actuators. The corresponding measured displacements of the mass $y^{m}(t)$ and $x^{m}(t)$ have been measured by a capacitive gauge, and are shown in Fig. 5(a). Fig. 5(b) shows the corresponding estimated trajectory of the mass $\left(y^{e}, x^{e}\right)$, calculated from the
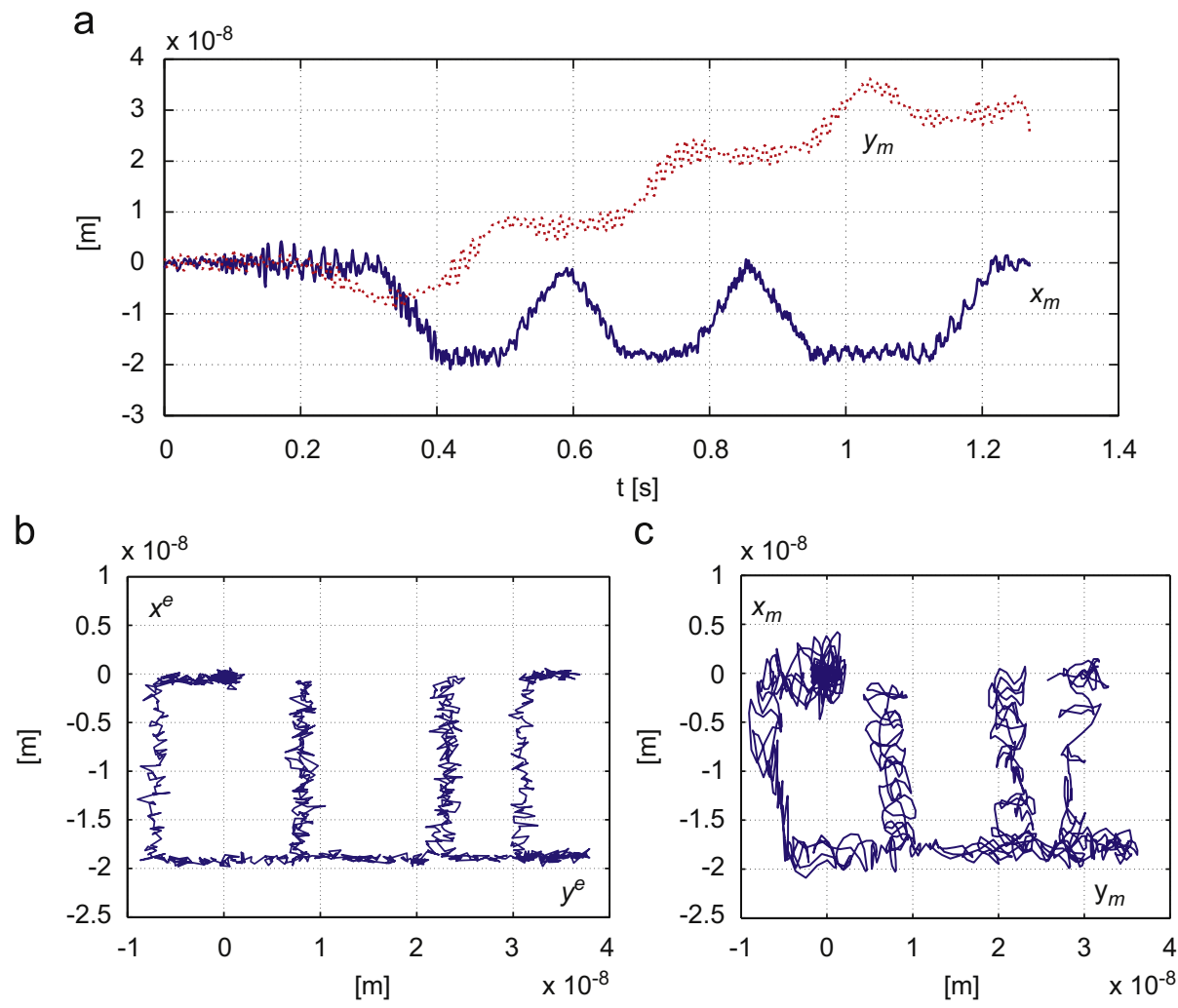

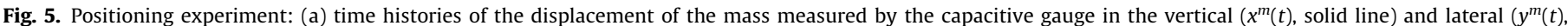

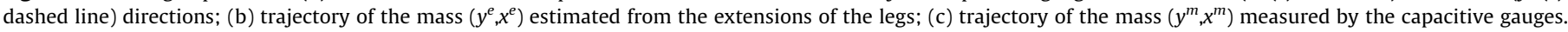


measured extensions of the legs $\left(q_{1}, q_{2}\right)$ as

$$
\left(\begin{array}{l}
y^{e} \\
x^{e}
\end{array}\right)=J^{-1}\left(\begin{array}{l}
q_{1} \\
q_{2}
\end{array}\right)
$$

Fig. 5(c) shows the resulting trajectory of the mass, i.e. $\left(y^{m}, x^{m}\right)$. Comparing with Fig. 5(b), one sees that the mass follows pretty well the motion requested by the actuators, with a precision of $\pm 2 \mathrm{~nm}$.

In the next section, the effect of the mechanical stabilization of the quadrupole on the beam jitter is evaluated using only one quadrupole, and an extremely simple model of the beam-based feedback.

\section{Effect of the quadrupole stability on the beam jitter}

At the scale of a single quadrupole, if $b(n)$ is the position of the pulse passing through the quadrupole at time $n$ and $x(n)$ is the vertical position of the quadrupole, we have

$b(n)=x(n)-k(n)$

where $k(n)$ is the corrector kick. A simple beam-based feedback system corrects the position of each pulse by subtracting the value measured at the previous one, i.e.

$k(n)=g_{p} b(n-1)$

where $g_{p}$ is the gain and $b(n-1)$ is the position of the previous pulse measured by the Beam Position Monitor (BPM) mounted on the quadrupole. Using the $Z$-transform, the transfer function $T_{B}(z)$ between the quadrupole and the beam can be expressed as

$T_{B}(z)=\frac{B(z)}{X(z)}=\frac{1}{1+g_{p} z^{-1}}$

where $B(z)$ and $X(z)$ are the $Z$-transform of $b(n)$ and $x(n)$. For a whole lattice of quadrupoles, this first order controller is sufficient to compensate for low frequency seismic excitations, because the spatial correlation of low frequency micro-seismic waves is excellent over several kilometers. However, at the scale of a single quadrupole, a more aggressive compensation is required at low frequency. In Ref. [21], a second order controller is given by

$T_{B}(z)=\frac{B(z)}{X(z)}=\frac{1-\left(g_{i}+g_{d 2}\right) z^{-1}+g_{d 2} z^{-2}}{1+\left(g_{p}+g_{d}-g_{i}-g_{d 2}\right) z^{-1}+\left(g_{d 2}-g_{d}\right) z^{-2}}$

where $g_{i}, g_{d}$ and $g_{d 2}$ are the gains of the controller.

Figs. 6(a) and (b) compare the transfer function of the mechanical stabilization in the lateral direction, $T_{S}^{L}$, and vertical direction, $T_{S}^{V}$, respectively, with $T_{B}(f)$ for the set of parameters: $g_{d 2}=1 ; \quad g_{p}=0.5 ; \quad g_{i}=1 ; \quad g_{d}=0.5$. Then, the transfer function between the ground and the beam is simply obtained by multiplying $T_{B}(f)$ and $T_{S}(f)$. The results are shown in Fig. 6(a) and (b) for the lateral and vertical directions.

Then, the power spectral density of the beam jitter is given by $\Phi_{b}^{B S}(f)=T_{B}(j \omega) T_{S}(j \omega) \Phi_{w}(\omega)\left[T_{B}(j \omega) T_{S}(j \omega)\right]^{*}$

where $\Phi_{w}(f)$ is the power spectral density of the ground motion and $T_{S}(j \omega)$ is the frequency transfer function from the ground to the quadrupole.

To evaluate the effect of the mechanical stabilization of the quadrupole on the beam jitter, we will assume that, when no stabilization is considered, the quadrupoles are rigidly bolted on the ground. In this case, Eq. (15) simplifies to

$\Phi_{b}^{B}(f)=T_{B}(j \omega) \Phi_{w}(\omega) T_{B}(j \omega)^{*}$ a
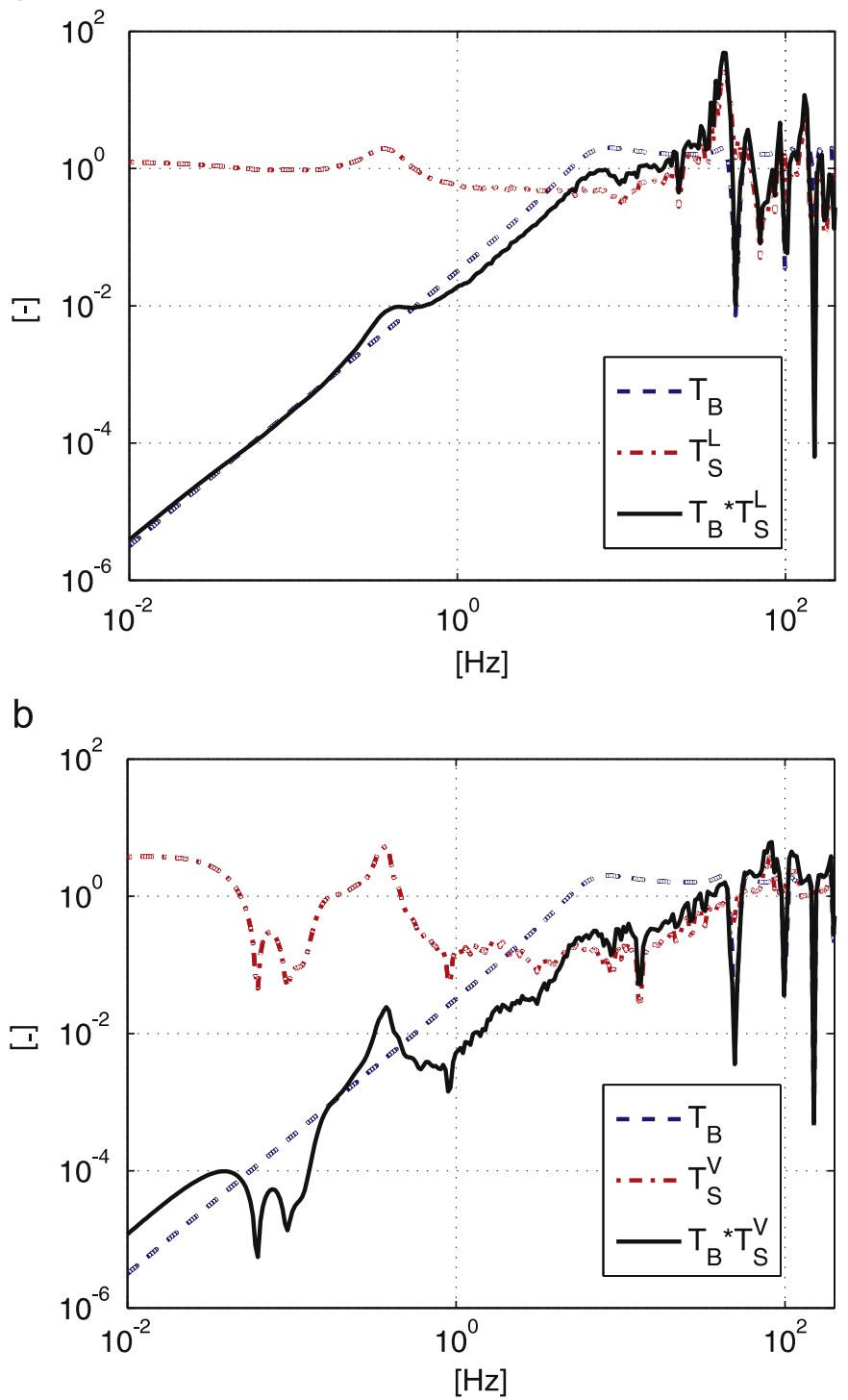

Fig. 6. Transfer function of the two d.o.f. test bench, combined with a second order strategy for the beam-based feedback (a) in the lateral direction; (b) in the vertical direction.

In both cases, the RMS value of the beam motion, $\sqrt{\left\langle b^{2}\right\rangle}$, is defined as

$\sqrt{\left\langle b^{2}\right\rangle}=\sqrt{\int_{0}^{\infty} \Phi_{b}(f) d f}$

The ground motion model used to evaluate $\Phi_{w}(\omega)$ is briefly explained hereafter.

A general expression of the ground motion model is given in Ref. [22] by a two-dimensional power spectral density $P(\omega, k)$, defined as

$P(\omega, k)=\frac{A}{\omega^{2} k^{2}}\left[1-\cos \left(L_{0} k\right)\right]+D(\omega) U(\omega, k)$

where

$U(\omega, k)=\frac{2}{\sqrt{k_{\max }^{2}-k^{2}}} \quad$ if $|k| \leq k_{\max }$

$U(\omega, k)=0 \quad$ if $|k|>k_{\max }$ 
$D(\omega)=\frac{a_{i}}{1+\left[d_{i}\left(\omega-\omega_{i}\right) / \omega_{i}\right]^{4}}$

and $L_{0}=B /\left(A \omega^{2}\right) . A, B, a_{i}, d_{i}$ and $v_{i}$ are the parameters of the model; $i=1, \ldots, n$ where $n$ is the number of waves propagating in the ground. The following coefficients have been found from measurements in the LHC tunnel: $i=1, A=10^{-4}\left(\mu \mathrm{m}^{2} \mathrm{~s}^{-1} \mathrm{~m}^{-1}\right) ; B=$ $10^{-4}\left(\mu \mathrm{m}^{2} \mathrm{~s}^{-3}\right) ; \omega_{1}=2 \pi * 0.14(\mathrm{rad} / \mathrm{s}) ; d_{1}=5 ; a_{1}=0.1\left(\mu \mathrm{m}^{2} / \mathrm{Hz}\right) ;$ $v_{1}=1000(\mathrm{~m} / \mathrm{s})[17]$.

As this model underestimates the effect of the technical noise, a simple model to take it into account has been proposed in Ref. [17],

$\Phi_{N}(f)=\frac{N_{0}}{1+\left(f / f_{0}\right)^{6}}$

where $f_{0}=4 \pi$ and $N_{0}$ is the amplitude of the filter. Based on measurements performed in the LHC tunnel, it has been identified that low and high bounds of the amplitude are $N_{0}=5 \times 10^{-3} \mathrm{~nm}^{2} / \mathrm{Hz}$ and $N_{0}=50 \mathrm{~nm}^{2} / \mathrm{Hz}$.

a

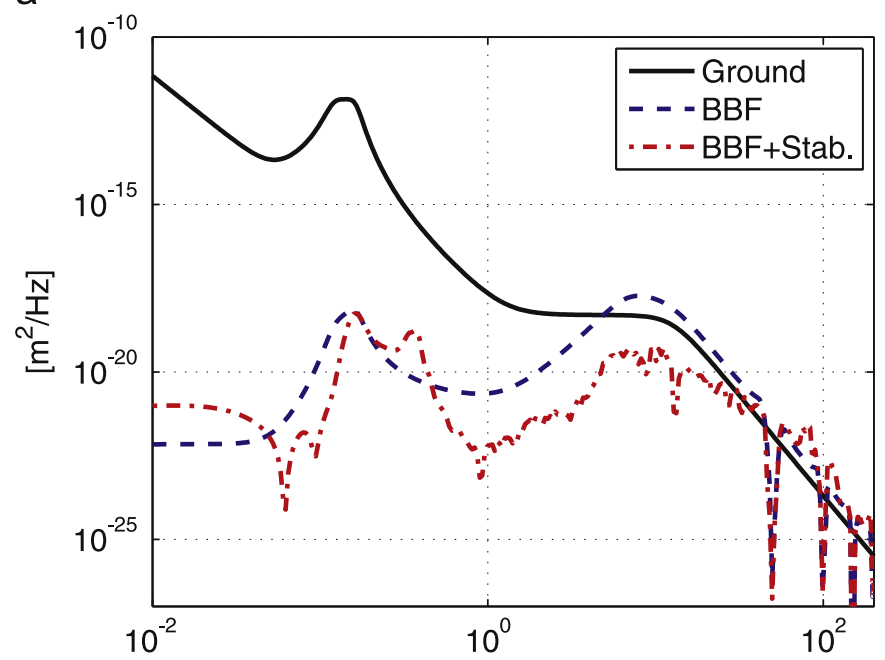

b

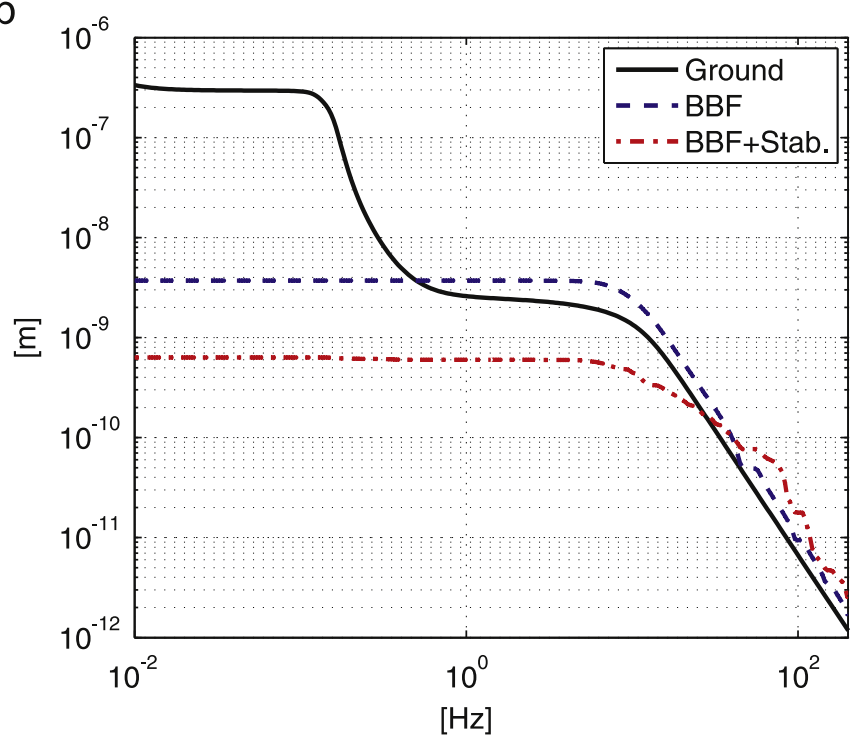

Fig. 7. (a) Power spectral densities in the vertical direction of the ground vibration $\Phi_{w}(f)$ (black solid line), of the beam jitter with beam-based feedback ON (dashed blue line) $\Phi_{b}^{B}(f)$, of the beam jitter with beam-based feedback ON and stabilization ON (dashed dotted red line) $\Phi_{b}^{B S}(f)$; integrated RMSs of the curves shown in (a). (For interpretation of the references to color in this figure legend, the reader is referred to the web version of this article.)

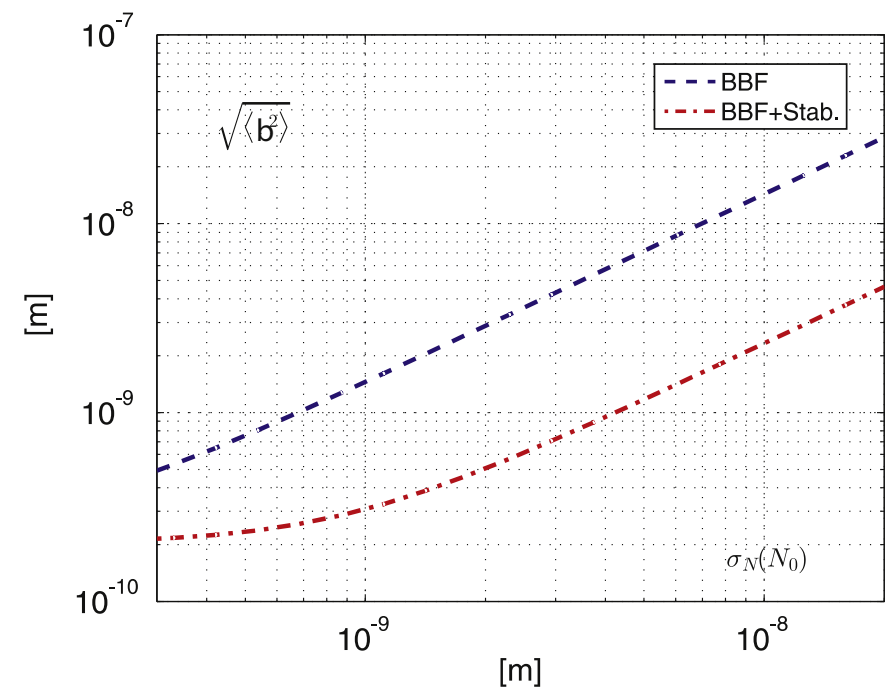

Fig. 8. RMS beam jitter for increasing level of technical noise and beam-based feedback (dashed blue line); beam-based feedback and stabilization (dashed dotted red line). (For interpretation of the references to color in this figure legend, the reader is referred to the web version of this article.)

Then, the power spectral density of a single point is obtained by integrating $P(\omega, k)$ over the wave number $k$, and adding the technical noise, i.e.

$\Phi_{w}(f)=\Phi_{N}(f)+\int_{0}^{\infty} P(\omega, k) \frac{d k}{2 \pi}$

Taking $N_{0}=0.5 \mathrm{~nm}^{2} / \mathrm{Hz}$ as the most representative level of technical noise in the LHC tunnel, Figs. 7(a) and (b) show, respectively, the power spectral density of the ground motion in the vertical direction $\Phi_{w}(f)$ (black solid line), and the corresponding integrated RMS. The figure also shows the power spectral density of the vertical beam jitter when beam-based feedback is turned ON (dashed blue line) $\Phi_{b}^{B}(f)$, and when both the beambased feedback and the mechanical stabilization are turned ON (dashed dotted red line) $\Phi_{b}^{B S}(f)$.

Using Eq. (17), the mechanical stabilization of the quadrupole reduces the RMS beam jitter from $\sqrt{\left\langle b^{2}\right\rangle}=4 \mathrm{~nm}$ to $\sqrt{\left\langle b^{2}\right\rangle}=$ $0.6 \mathrm{~nm}$. From Eq. (20), one sees that these values depend on the level of the technical noise. To evaluate the acceptable level of technical noise, let us now take $N_{0}$ as a variable and calculate the RMS beam jitter with and without mechanical stabilization. The results, shown in Fig. 8, indicate that, above $N_{0}=1 \mathrm{~nm}$, the mechanical stabilization reduces the beam jitter by roughly a factor 10 .

\section{Conclusions}

In this paper, it has been shown that the same active mounts can be used to support the quadrupoles of future particle colliders, and comply with all the requirements. The concept has been validated experimentally on a test bench consisting of a heavy mass mounted on two active legs. Using a control law based on the measurement of the relative displacement between the mass and an inertial reference, it has been shown that, in the frequency range between 1 and $20 \mathrm{~Hz}$, the active mounts reduce the transmission of the ground vibration by a factor 10 in the vertical direction, and by a factor 2 in the lateral direction. During the day, at $1 \mathrm{~Hz}, \sigma_{x}(f)$ is reduced from 5.7 to $2.1 \mathrm{~nm}$, i.e. a reduction by a factor 2.5 . During the night, when the ground motion is even lower, $\sigma_{x}$ is reduced from 2 to $0.9 \mathrm{~nm}$, at $1 \mathrm{~Hz}$, i.e. below the requested value of $1.5 \mathrm{~nm}$. 
Then, in open loop configuration, it has been shown that the active mounts can also provide the requested positioning capability in both vertical and lateral directions. Finally, the effect of the mechanical stabilization of the quadrupole on the beam jitter has been investigated. Using a simple model for the beam-based feedback, it has been estimated that the stabilization reduces the beam jitter by a factor 10 . In other words, the stabilization relaxes the acceptable level of technical noise by a factor 10 .

In a future work, the strategy to support the quadrupole will be extended to a six d.o.f. slender and heavy electromagnet of $400 \mathrm{~kg}$. This involves to design a guide to remove the spurious d.o.f., to take the flexibility of the quadrupole into account, and to extend of the controller. Better results are also expected from a more adapted sensor.

\section{Acknowledgments}

The research leading to these results has been jointly funded by the European Commission under the FP7 Research Infrastructures project EuCARD, Grant agreement no. 227579, and by the Brain Back to Brussels program from Brussels Capital Region for the first author. The authors also gratefully acknowledge Daniel Schulte for useful discussions on the beam dynamics, and the reviewer for their valuable comments.

\section{References}

[1] J.P. Delahaye, Towards CLIC feasibility, in: IEEE International Particle Accelerator Conference IPAC10, 23-25 May 2010, Kyoto, Japan, 2010.
[2] C. Collette, K. Artoos, A. Kuzmin, S. Janssens, M. Sylte, M. Guinchard, C. Hauviller, Nuclear Instruments and Methods in Physics Research A 621 (1-3) (2010) 71.

[3] J. Spanos, Z. Rahman, G. Blackwood, A soft 6-axis active vibration isolator, in: Proceedings of the American Control Conference, Seatle, Washington, 1995.

[4] A. Hanieh, Active isolation and damping of vibrations via Stewart platform, Ph.D. Thesis, University of Brussels, 2004.

[5] A. Preumont, Vibration Control of Active Structures: An Introduction, second ed., Kluwer Academic Publishers, Dordrecht (The Netherlands), 2002.

[6] 〈http://clic-stability.web.cern.ch/clic-stability/ $\rangle$.

[7] D. Schulte, Beam based alignment in the new CLIC main linac2, in: IEEE Particule Accelerator Conference 2009 PAC09, 4-8 May 2009, Vancouver, Canada, 2009.

[8] D. Schulte, CLIC stabilisation meeting 1: Specification for stabilisation, March 2008.

[9] C. Montag, Nuclear Instruments and Methods in Physics Research A 378 (1996) 369.

[10] C. Montag, Active stabilization of mechanical quadrupole vibrations in a linear collider test facility, Ph.D. Thesis, Hamburg University, 1996

[11] P.R. Saulson, Review of Scientific Instruments 55 (8) (1984) 1315.

[12] P.G. Nelson, Review of Scientific Instruments 62 (9) (1991) 2069

[13] M.J. Vervoordeldonk, T.A.M. Ruijl, R.M.G. Rijs, Development of a novel active isolation concept, in: ASPE Spring Topical Meeting, 2004.

[14] M.J. Vervoordeldonk, H. Stoutjesdijk, Recent developments, a novel active isolation concept, in: 6th Euspen International Conference, Baden bei Wien, 2006.

[15] M.J. Vervoordeldonk, T.A.M. Ruijl, R.M.G. Rijs, J.C.A. Muller, Actuator arrange for active vibration isolation comprising an inertial reference mass, Technical Report Patent Number: US 2007/0035074 A1, United States Patent, 2007.

[16] K. Kar-Leung Miu, A low cost, DC-coupled active vibration system, Ph.D. Thesis, Massachusetts Institute of Technology, September 2008.

[17] C. Collette, K. Artoos, M. Guinchard, C. Hauviller, Physical Reviews Special Topics-Accelerators and Beams 13 (2010) 072801.

[18] Physik Instrumente catalogue.

[19] Guralp System Limited catalogue.

[20] National Instruments catalogue.

[21] D. Schulte, C. Collette, J. Pfingstner, A. Jeremie, CLIC stabilisation meeting 8: some comments on feedback and feedforward at the IP, December 2009.

[22] A. Sery, O. Napoly, Physical Review E 53 (5) (1996) 5323. 